\title{
Factores asociados al síndrome de burnout en docentes de colegios de la ciudad de Cali, Colombia *
}

\author{
Factors associated to Burnout Syndrome in high \\ school teachers in Cali, Colombia
}

Recibido: julio 12 de 2010 | Revisado: septiembre 13 de 2010 | Aceptado: octubre 8 de 2010

\author{
FÁtima DíAz BAMBUla ** \\ ANA MARÍA LÓPEZ SÁNCHEZ *** \\ María Teresa Varela ARÉvalo \\ Pontificia Universidad Javeriana, Cali, Colombia
}

SICI: 2011-2777(201203)11:1<217:FASBDC>2.0.CO;2-X

Para citar este artículo: Díaz, F., López, A. \& Varela, M. T. (2012). Factores asociados al síndrome de burnout en docentes de colegios de la ciudad de Cali, Colombia. Universitas Psychologica, 11(1), 217227.

* Trabajo de grado de la carrera de Psicología, adscrito al grupo de investigación Salud y Calidad de Vida de la Pontificia Universidad Javeriana de Cali.

** AA. 25 122, Unicentro, Cali, Colombia. E-mail: diazbambulafatima@gmail.com

*** Calle 18 \# 118-250. Vía Pance. Oficina 3-49 Ed. El Samán,Cali,Colombia.E-mails: anamarial@javerianacali.edu.co,mtvarela@javerianacali.edu.co

\section{RESUMEN}

El objetivo de la investigación fue establecer los factores asociados al síndrome de burnout en docentes de dos instituciones educativas formales privada y pública de la ciudad de Cali, Colombia. Se describieron las dimensiones del síndrome (Agotamiento Emocional, Despersonalización y Falta de Realización Personal) y su relación con los factores organizacionales, el Estrés del Rol y las características sociodemográficas de los docentes. Para esto se aplicó el Cuestionario de Burnout en Profesores - Modificado (CBP-M) a una muestra de 82 docentes de un colegio público y uno privado. Los resultados muestran bajos niveles de burnout en ambas instituciones. Respecto a los factores asociados, se halló relación con el Estrés de Rol y los factores organizacionales de Supervisión, Condiciones Organizacionales y Preocupaciones Profesionales. Las variables sociodemográficas no presentaron relaciones significativas con el síndrome, a excepción del nivel de enseñanza en el cual los docentes imparten clases.

Palabras clave autores:

Síndrome de burnout, docentes, factores organizacionales, estrés de rol, variables sociodemográficas.

Palabras clave descriptores:

Cuestionario de Burnout en Profesores (CBP-M), psicología organizacional, investigación cuantitativa.

\section{A B S T R A C T}

The goal of the study was to locate factors related to burnout syndrome in teachers of two formal private and public educational institutions from Cali, Colombia. The syndrome's dimensions (Emotional Exhaustion, Depersonalization and Reduced Sense of Personal Accomplishment) and their relation to organizational factors, the Stress of the Role and sociodemographic characteristics of the teachers were described. The Modified Burnout in Teachers Questionnaire (CBP-M) was applied to a sample of 82 teachers from one public and one private school. Results show a low level of burnout syndrome in both institutions. An association with the levels of stress of role and organizational factors such as supervision, job conditions and professional worries was found. The socio-demographic characteristics did not show significant relations with the syndrome, with the exception of the level of education in which the teachers give classes.

Key words authors:

Burnout syndrome, teachers, organizational factors, stress of role, sociodemographic characteristics.

Key words plus:

Modified Burnout in Teachers Questionnaire (CBP-M), organizational psychology, quantitative research. 


\section{Introducción}

Actualmente, múltiples estudios muestran evidencia de la presencia del síndrome de burnout en personas con profesiones de ayuda, entre ellas la docencia. Esta labor presenta una alta vulnerabilidad al burnout, debido a la realización simultánea de múltiples actividades y funciones que implican asegurar que los estudiantes reciban una educación de calidad (Alderete, Pando, Aranda \& Balcázar, 2003; García, 1996 citado por Matud, García \& Matud, 2002; Moreno-Jiménez, Garrosa \& González, 2000; Ponce, Bulnes, Aliaga, Atalaya $\&$ Huertas, 2005), así como exigencias grandes y conflictivas, no solo por parte de los alumnos, sino también de las familias, las redes sociales y la administración (Anadón, 2005; Aranda, Pando \& Pérez, 2004; Alderete et al., 2003; Bórquez, 2004; García, 1996 citado por Matud et al., 2002; Ponce et al., 2005).

El síndrome de burnout consiste en una respuesta psicológica al estrés laboral crónico que compromete tres dimensiones: Agotamiento Emocional, Despersonalización y Pérdida de Logro o Realización Personal, todas ellas referidas a la relación entre el individuo y el ámbito laboral (Maslach \& Jackson, 1979, 1999 citados por Hernández \& Olmedo, 2004), compuesto por un conjunto de síntomas físicos, psicológicos y sociales como resultado del desequilibrio entre los esfuerzos realizados por el trabajador y los resultados obtenidos, los cuales no satisfacen las expectativas del profesional (De la Peña et al., 2002). El burnout se genera mediante un proceso que evoluciona lentamente y puede ser desconocido para el mismo sujeto que lo padece (Moreno-Jiménez \& Peñacoba, 1999).

El Agotamiento Emocional es la sensación de cansancio, fatiga, vacío; la persona se encuentra gastada, sin energía ni recursos emocionales y no puede dar más de sí. La Despersonalización consiste en la adopción de una actitud de distanciamiento, cinismo y endurecimiento emocional, con tendencia a considerar o percibir a las personas como objetos insensibles, generando una notable pérdida de la capacidad de contacto interpersonal, insensibilidad y falta de empatía hacia los otros. La
Pérdida de Logro o Realización Personal implica un sentimiento de disminución de la competencia, de manera que el profesional se percibe cada vez más ineficaz e inútil, la autoestima disminuye progresivamente y como consecuencia se limita cada vez más la capacidad para desarrollar con éxito el rol profesional, este sentimiento de insatisfacción e infelicidad con el propio rendimiento laboral se puede extender también a los ámbitos de la vida privada (Maslach \& Jackson, 1979, 1999 citados por Hernández \& Olmedo, 2004).

En Colombia, algunas investigaciones han evidenciado la relación del síndrome de burnout con la docencia. En un estudio con docentes oficiales en la ciudad de Medellín se identificó que el $46.8 \%$ de ellos presentaban burnout o estaban en alto riesgo de presentarlo. En otro estudio en Ibagué, los resultados señalan que gran parte de los docentes de la muestra presentaba un nivel de burnout bajo, pero se destacaba el hecho de que el $34 \%$ presentaran niveles altos en cinismo (o Despersonalización) y el $31 \%$ reportaran un alto grado de ineficiencia en el trabajo, las cuales son características importantes del síndrome de burnout (Hermosa, 2006; Restrepo-Ayalal, Colorado \& Cabrera, 2005). Además, un estudio en Bogotá encontró una prevalencia del síndrome del $15.6 \%$ en docentes de colegios públicos y porcentajes altos en los factores asociados relacionados con el entorno laboral (18.96 \%) y la satisfacción personal (16.34 \%).

Entre los modelos que se han propuesto para explicar el síndrome de burnout y su evolución, se encuentra el de Leithwood, Menzies, Jantzi y Leithwood (1999 citados por Moriana \& Herruzo, 2004), que plantea que la estimación y las estrategias frente a las demandas laborales que el docente realiza en su trabajo, son determinantes en la aparición del burnout, dándose más importancia a las características organizacionales. Este modelo parte de tres constructos interrelacionados: las transformaciones en la escuela; las decisiones de los administradores y jefes de las instituciones educativas y los factores organizacionales y personales.

Las investigaciones realizadas sobre el síndrome de burnout en docentes han señalado algunos 
factores relacionados con su aparición, los cuales pueden agruparse en variables organizacionales y propias del trabajo, sociodemográficas y de personalidad (Moriana \& Herruzo, 2004).

En relación con las variables organizacionales y propias del trabajo, se han planteado como factores asociados el clima laboral, la satisfacción y sobrecarga laboral, los trabajos administrativos, las clases con muchos alumnos, el conflicto de rol, los problemas con los superiores, compañeros y padres de alumnos, las legislaciones educativas, el desarrollo profesional deficitario, los salarios bajos, los trastornos de conducta y conductas disruptivas de los alumno (Alderete et al., 2003; Bergadá, Neudeck, Parquet, Tisiotti \& Dos Santos, 2005; Hermosa, 2006; Moreno-Jiménez, Arcenillas, Morante \& Garrosa, 2005; Ponce et al., 2005; Restrepo-Ayalal et al., 2005; Tisiotti, Parquet \& Neudeck, 2007).

En relación con las variables sociodemográficas no existe mucha consistencia en los resultados encontrados, mencionándose como posibles variables asociadas el sexo, la edad y el estado civil (Alderete et al., 2003; Hermosa, 2006; Moreno, Aranda, Aldrete, Flores \& Pozo, 2006; Ponce et al., 2005; Restrepo-Ayalal et al., 2005; Tisiotti et al., 2007). Entre las variables de personalidad se han relacionado el locus de control externo, la autoconciencia, el autocontrol, la autoeficacia, la personalidad resistente, el patrón de conducta tipo A, la autoestima, los pensamientos irracionales, el neuroticismo, la empatía, el optimismo, la extroversión, la rigidez y la depresión (Bermejo-Toro \& Prieto-Usúa, 2006; Hernández, Olmedo \& Ibáñez, 2004; Moreno-Jiménez et al., 2005; Ortega \& López, 2003).

Teniendo en cuenta que la evidencia sobre los factores potencialmente asociados al burnout no es aún concluyente y que en Colombia, particularmente en la ciudad de Cali, se han hecho pocas investigaciones sobre el tema, se realizó este estudio con el propósito de identificar la presencia del síndrome $y$ los factores asociados a este en docentes de un colegio público y uno privado de esta ciudad, estableciendo una comparación entre ambos tipos de institución.

\section{Método}

\section{Tipo de investigación}

La investigación fue no experimental con diseño transversal-correlacional (Hernández, Fernández \& Baptista, 1998).

\section{Participantes}

La muestra estuvo conformada por 82 docentes, 44 de un colegio privado y 38 de uno público. Los criterios de selección de los docentes de ambas clases de colegio fueron: enseñar en cursos de básica primaria o secundaria y no tener antecedentes de trastornos psiquiátricos diagnosticados. La muestra fue seleccionada de manera intencional.

\section{Instrumento}

Se utilizó el Cuestionario de Burnout en Profesores-Modificado (CBP-M) construido para la investigación con base en el Cuestionario de Burnout en Profesores Revisado (CBP-R) de Moreno-Jiménez et al. (2000) y evaluado por 3 jueces expertos. El CBP-M está compuesto por 70 ítems que evalúan indicadores de burnout en docentes, con respecto a las dimensiones de Agotamiento Emocional, Despersonalización y Falta de Realización. Adicionalmente, evalúa las variables de tipo organizacional y laboral que pueden actuar como desencadenantes de estos procesos: estrés del rol (preguntas referentes al proceso de estrés, específicamente como producto de las disfunciones del rol), desorganización (condiciones en las que se realiza el trabajo -materiales y recursos de los que se dispone, estilo de dirección y apoyo recibido por parte del supervisor-), problemática administrativa (preocupaciones profesionales y reconocimiento profesional que perciben de su profesión). 


\section{Procedimiento}

Una vez obtenido el permiso para la realización de la investigación en los colegios, se presentaron los objetivos a los docentes. Los que aceptaron participar firmaron el consentimiento informado. La aplicación del instrumento se realizó de manera grupal y tuvo una duración promedio de una (1) hora.

\section{Análisis de resultados}

Para el análisis de los datos se utilizó el Paquete Estadístico para las Ciencias Sociales: SPSS versión 16 para Windows. Inicialmente, se describieron las características sociodemográficas de los docentes evaluados, tanto para el colegio privado como para el público. Se establecieron tres niveles de burnout, a partir de la clasificación de los puntajes en severo, moderado y normal. Para establecer los factores asociados al burnout (tanto para el puntaje total como para cada una de las tres dimensiones) se utilizó la prueba estadística de chi cuadrado.

\section{Resultados}

\section{Características de la muestra}

En total se evaluaron 44 docentes de un colegio privado y 38 de un colegio público, de la ciudad de Cali. En el colegio privado, el $52 \%$ de los docentes era hombre, mientras que en el colegio público la mayor parte de la muestra estaba constituida por mujeres (71 \%). La edad promedio de los docentes en el colegio privado fue de 37 años $(D E=9.6$, rango 23-63) y en el colegio público fue de 46 años $(D E=6.8$, rango 34-60).

La mayoría de docentes tenían pareja habitual estable (72\% en el colegio privado y $62 \%$ en el colegio público). En cuanto a la situación laboral se encontró que el 100 \% de los docentes del colegio privado tienen un tipo de contratación a término fijo, mientras que en el colegio público el $97 \%$ de los docentes presentaron un tipo de contratación a término indefinido. Con respecto al nivel de enseñanza en el que los docentes imparten clases, se encontró que en el colegio privado hay un $34 \%$ en educación primaria, un $43 \%$ en educación secundaria y el $23 \%$ en ambos niveles. En el colegio público se encontró que el $34 \%$ imparten clases en el nivel de educación primaria y el $66 \%$ en educación secundaria. El promedio de años de experiencia en la labor docente en el colegio privado fue de 14 años $(D E=7.8)$ y en el colegio público de 23 años $(D E=6.9)$. El mayor número de materias dictadas por los docentes del colegio privado fue de 5 y el mínimo de $1(M=1$; $D E=0.8)$, y en el colegio público el máximo número fue de 10 y el mínimo $1(M=4 ; D E=2.8)$.

\section{Síndrome de burnout}

Con relación al síndrome de burnout se encontró que, en el colegio privado, el $15 \%$ de los docentes presentaba un nivel moderado y el resto se encontraba en nivel normal. Por su parte, en el colegio público, el $22 \%$ de los docentes estaba en nivel moderado y los demás en nivel normal (Tabla 1).

TABLA 1

Niveles de burnout en docentes de colegios públicos y privados

\begin{tabular}{lccccccc}
\hline & \multicolumn{3}{c}{ Colegio público } & \multicolumn{3}{c}{ Colegio privado } & \multirow{2}{*}{ Valor $p^{*}$} \\
\hline Síndrome burnout & $85 \%$ & $15 \%$ & $0 \%$ & $78 \%$ & $22 \%$ & $0 \%$ & 0.322 \\
Agotamiento Emocional & $57 \%$ & $43 \%$ & $0 \%$ & $68 \%$ & $32 \%$ & $0 \%$ & 0.407 \\
Despersonalización & $98 \%$ & $2 \%$ & $0 \%$ & $89 \%$ & $11 \%$ & $0 \%$ & 0.142 \\
Falta de Realización Personal & $79 \%$ & $21 \%$ & $0 \%$ & $67 \%$ & $33 \%$ & $0 \%$ & 0.181 \\
\hline
\end{tabular}

* Prueba $\mathrm{Chi}^{2}$

Fuente: elaboración propia. 
Al comparar los resultados del nivel del síndrome de burnout total entre ambos tipos de colegio, se encuentra que no existen diferencias estadísticamente significativas entre los docentes del colegio público y privado $\left(\chi^{2}=0.576 ; p=0.322\right)$.

Al comparar el nivel total de burnout con los factores sociodemográficos, se encontró que no existen diferencias estadísticamente significativas entre hombres y mujeres, entre quienes tienen y no tienen pareja habitual, entre quienes tienen menos o más de 20 años de experiencia, entre quienes tienen o no hijos; tampoco existe relación con el número de materias que imparten los docentes en la institución, la situación laboral, enseñar en primaria, bachillerato o en ambos niveles de enseñanza.

$\mathrm{Al}$ analizar por separado las dimensiones del síndrome de burnout, se encontró respecto al Agotamiento Emocional, que en el colegio privado el $38 \%$ de los docentes presentan un nivel moderado y los demás se encuentran en un nivel normal. En el colegio público el 43 \% de los docentes están en nivel moderado de Agotamiento Emocional y los demás presentan un nivel normal.

$\mathrm{Al}$ analizar individualmente los indicadores de Agotamiento Emocional, se encontró que la mayoría de docentes de ambas instituciones refieren no sentirse agotados por enseñar (72\%), no consideran que su trabajo afecte negativamente sus relaciones sociales $(85 \%)$ y refieren no sentirse deprimidos a causa de la profesión (80 \%).

Se encontró una relación estadísticamente significativa entre los niveles de Agotamiento Emocional y los niveles de enseñanza, hallándose que los docentes de bachillerato son más vulnerables a presentar agotamiento $\left(\chi^{2}=4.91 ; p=0.038\right)$. En cuanto al tipo de colegio, se encuentra que no existen diferencias estadísticamente significativas en los niveles de Agotamiento Emocional en los docentes del colegio público ni en los del colegio privado.

En la dimensión de Despersonalización, el $2 \%$ de los docentes del colegio privado están en nivel moderado y el resto en nivel normal; mientras que en el colegio público se encontró un 11 \% en nivel moderado y los demás en nivel normal.
$\mathrm{Al}$ analizar los indicadores de Despersonalización, se halló que la mayoría de los docentes indican no sentir a sus alumnos como enemigos (93\%), no tienden a tratar a los estudiantes como objetos insensibles (92\%), tratan a sus estudiantes cálidamente (94\%), sus estudiantes no le fastidian (97\%), perciben ser agradables para sus estudiantes (87\%), les preocupa lo que le suceda a sus alumnos (99\%) y no se definen como profesores por obligación (94\%).

En la comparación de los niveles de Despersonalización con los factores sociodemográficos, no se encontraron diferencias estadísticamente significativas en ninguna de estos. De igual manera, no se encuentran diferencias estadísticamente significativas entre los docentes del colegio público y privado.

En relación con la Falta de Realización Personal, en el colegio privado se encuentra un $33 \%$ de los docentes con un nivel moderado y los demás en nivel normal. En el colegio público, el $21 \%$ de los docentes están en nivel moderado y los demás docentes en nivel normal. Al examinar los indicadores de Falta de Realización Personal se encontró que los docentes, en su mayoría, no se sienten contentos con su trabajo, no disfrutan suficientemente su vida laboral y no la consideran provechosa, no volverían a ser docentes y sienten que pueden producir cambios positivos en sus alumnos.

En la comparación de los niveles de Falta de Realización Personal con los factores sociodemográficos no se encontraron diferencias estadísticamente significativas en ninguno. De igual manera, no se encuentran diferencias estadísticamente significativas entre los docentes del colegio público y privado.

En cuanto a los factores organizacionales, se encontró relación entre el burnout con la Supervisión $(p=0.02)$ y las Condiciones Organizacionales $(p=0.048)$; a su vez se halló relación entre el Agotamiento Emocional, la Supervisión, $(p=0.04)$, las Condiciones Organizacionales, $(p=0.01)$ y las Preocupaciones Profesionales $(p=0.01)$. Asimismo, el Estrés de Rol se relacionó con el síndrome de burnout $(p=0.00)$, el Agotamiento Emocional $(p=0.00)$ y la Falta de Realización Personal ( $p=0.02$ ) (Tablas 2 y 3 ). 
TABLA 2

Factores organizacionales en docentes de colegios públicos y privados

\begin{tabular}{lcccccc}
\hline & \multicolumn{3}{c}{ Colegio público } & \multicolumn{3}{c}{ Colegio privado } \\
& Normal & Moderado & Severo & Normal & Moderado & Severo \\
\hline Estrés de Rol & $44 \%$ & $53 \%$ & $3 \%$ & $61 \%$ & $39 \%$ & $0 \%$ \\
Condiciones Organizacionales & $81 \%$ & $19 \%$ & $0 \%$ & $63 \%$ & $37 \%$ & $0 \%$ \\
Supervisión & $57 \%$ & $43 \%$ & $0 \%$ & 64 & $36 \%$ & $0 \%$ \\
Falta de Realización Profesional & $18 \%$ & $79 \%$ & $3 \%$ & $19 \%$ & $79 \%$ & $2 \%$ \\
Preocupaciones Profesionales & $53 \%$ & $0 \%$ & $47 \%$ & $41 \%$ & $0 \%$ & $59 \%$ \\
\hline
\end{tabular}

Fuente: elaboración propia.

TABLA 3

Relación entre niveles de burnout y factores organizacionales

\begin{tabular}{lcccc}
\hline & $\begin{array}{c}\text { Síndrome de } \\
\text { burnout }\end{array}$ & $\begin{array}{c}\text { Agotamiento } \\
\text { Emocional }\end{array}$ & Despersonalización & $\begin{array}{c}\text { Falta deRealización } \\
\text { Personal }\end{array}$ \\
\hline Estrés de rol & 0 & 0 & 0.14 & 0.02 \\
Condiciones Organizacionales & 0.048 & 0.01 & 0.639 & 0.355 \\
Supervisión & 0.02 & 0.04 & 0.576 & 0.538 \\
Falta de Realización Profesional & 0.086 & 0.63 & 0.088 & 0.32 \\
Preocupaciones Profesionales & 0.024 & 0.01 & 0.20 & 0.087 \\
\hline
\end{tabular}

* Prueba de $\mathrm{Chi}^{2}$

Fuente: elaboración propia.

\section{Discusión}

El estudio tuvo como objetivo identificar indicadores del síndrome de burnout en los docentes de instituciones educativas formales privadas y públicas de la ciudad de Cali y establecer los factores asociados a éstos. Los factores evaluados fueron de tipo organizacional, características sociodemográficos y Estrés de Rol.

Según los resultados, no se encontraron niveles severos de burnout en los docentes del colegio público y privado, aunque sí se presentaron algunos indicadores moderados del síndrome en ambas instituciones. En cuanto a las dimensiones del síndrome, se observa que la mayoría de los docentes, tanto del colegio público como del privado, presentan niveles normales de Agotamiento Emocional, Despersonalización y Falta de Realización Personal, y un porcentaje bajo presentan niveles moderados de estos. Los indicadores del síndrome de burnout y sus tres dimensiones no presentaron relaciones significativas con el tipo de colegio en el que los docentes trabajan.

En cuanto a las variables sociodemográficas y la relación con el síndrome de burnout y sus tres dimensiones (Agotamiento Emocional, Despersonalización y Falta de Realización Profesional), no se encontraron relaciones significativas con las variables edad, sexo, relaciones personales, número de hijos, experiencia laboral, número de materias y años de experiencia. Al respecto, se han encontrado diferentes resultados en las investigaciones que intentan relacionar estas variables (Albanesi, De Bortoli \& Tifner, 2006; Hermosa, 2006; Moreno et al., 2006; Moreno-Jiménez et al., 2005; Ortega \& López, 2003; Ponce et al., 2005). La ausencia de relación entre el burnout y las variables sociodemográficas en este estudio, podría explicarse por el hecho de que las características propias de los docentes que son 
externas a su labor, como el número de hijos, la edad y el sexo, han sufrido transformaciones sociales importantes, de manera que los roles cada vez van siendo más compartidos entre hombres y mujeres, la cantidad de hijos cada vez es menor y el rango de edad para el trabajo cada vez es más amplio, semejante a lo encontrado por Moriana y Herruzo (2004) en su meta-analisis donde se muestra resultados poco concluyentes sobre esta asociación; por lo cual se llega a la conclusión de que probablemente el burnout tiene más relación con las variables organizacionales que con las características propias del sujeto.

Por otra parte, teniendo en cuenta los factores organizacionales evaluados, la Falta de Realización Profesional, las Preocupaciones Profesionales, la Supervisión y las Condiciones Organizacionales, se encontró que el síndrome de burnout presenta relación significativa con las condiciones organizacionales y la supervisión. Al respecto, donde se encontró relación con el burnout fue en los niveles de enseñanza, específicamente en la dimensión de Agotamiento Emocional, encontrándose mayor incidencia en los docentes que imparten clases en secundaria, seguido por los que imparten clases en ambos niveles (primaria y secundaria). Esto es diferente de lo encontrado en el meta-análisis de Moriana y Herruzo (2004), quienes encuentran que el nivel de enseñanza tendría que ver con las dimensiones de Despersonalización y Falta de Realización Personal, pero no con Agotamiento Emocional.

Lo anterior podría estar relacionado con la etapa del ciclo de desarrollo de los estudiantes con los cuales tienen contacto los docentes de secundaria, que generalmente son adolescentes; en esta etapa, según Baró (s. f.), las personas alcanzan un nivel de abstracción que les permite hacer cuestionamientos relacionados con los padres, la familia y los adultos en general, y esto implica que los docentes enfrenten constantemente cuestionamientos sobre su labor.

A su vez, y teniendo en cuenta las condiciones organizacionales en las que los docentes realizan su trabajo, en términos de los materiales y recursos de los que disponen, así como las relacionadas con el estilo de dirección y apoyo recibido por parte del supervisor (supervisión), podría pensarse que la relación encontrada posiblemente es debida a que el síndrome es el resultado del estrés laboral crónico, lo que de alguna manera se relaciona con las condiciones que desde el trabajo se le brindan al docente. Esto coincide con lo planteado por Moreno-Jiménez et al. (2000), quienes definen estos factores organizacionales como las condiciones en las que se realiza el trabajo, el estilo de dirección y el apoyo recibido por parte del supervisor.

Asimismo, se encontró una relación significativa entre el burnout y la variable estrés de rol, independientemente del tipo de colegio (público y privado). Esto podría estar relacionado con que, en general, en cualquier tipo de institución, la docencia es una profesión con alta vulnerabilidad frente al estrés laboral, dadas las múltiples funciones de las profesiones de ayuda. Dichas profesiones giran en torno a garantizar el bienestar de otros, y en el caso de la docencia, en los estudiantes, asegurándose de que reciban una educación de calidad, tal como lo plantean Alderete et al. (2003), Byrne (1999), García (1996 citado por Matud et al., 2002), Moreno-Jiménez et al. (2000) y Ponce et al. (2005).

En cuanto al Agotamiento Emocional, se encuentra que más de un tercio de los docentes está en nivel moderado de esta dimensión en ambas instituciones, lo que puede explicarse por una posible sobrecarga laboral, por las situaciones administrativas, el nivel de escolaridad de los docentes y la satisfacción laboral. De igual manera, podría deberse a que las expectativas de los docentes en relación con su labor, posiblemente no concuerdan con lo que experimentan en su trabajo.

De acuerdo a los hallazgos encontrados, se evidencia una relación significativa con los factores organizacionales que tiene que ver con las Preocupaciones Profesionales, Supervisión y Condiciones Organizacionales. La relación entre el agotamiento y el estilo de dirección y apoyo de parte del supervisor (supervisiones), así como las condiciones en las que se realiza el trabajo relacionado con los recursos y materiales (condiciones organizacionales), podría deberse a que 
esta sensación de cansancio está ligada a las condiciones que el ámbito laboral le ofrece a los docentes. Entre estas condiciones se encuentran los materiales, el estilo de dirección y el apoyo de parte de la institución.

Asimismo, la relación significativa que existe entre esta dimensión del síndrome con las preocupaciones profesionales, podría deberse al cansancio emocional, a la sensación de no poder dar más de sí en su desempeño laboral, lo que genera las preocupaciones profesionales por la propia valoración que hace el mismo docente de su situación y de sus logros.

Además de lo anterior, se encuentra una relación significativa entre el Agotamiento Emocional y el Estrés de Rol, lo cual coincide con la investigación de Moreno-Jiménez, Garrosa y González (1999). La relación entre el Estrés de Rol y estas dimensiones del burnout puede entenderse si se tiene en cuenta que el estrés se caracteriza porque los docentes perciben que las estrategias utilizadas por ellos no son suficientes para enfrentar las demandas laborales, de acuerdo a lo que plantean Matud et al. (2002), Trucco y Valenzuela (1999) y Ponce et al. (2005). Lo anterior puede llevar al agotamiento emocional, que se caracteriza por que la persona se encuentra gastada emocionalmente y no puede dar más de sí.

En cuanto a la dimensión de Falta de Realización Personal, se encontró que pocos de los docentes de ambos colegios se ubican en un nivel moderado, lo cual concuerda con los estudios realizados por Alderete et al. (2003), Hermosa (2006), Pando et al. (2006), Restrepo-Ayalal et al. (2005) y Tisiotti et al. (2007). Esto podría ser explicado debido a que la mayoría de los docentes se encuentran satisfechos con su trabajo, sienten que pueden producir cambios positivos en los estudiantes, pero sin embargo refieren no disfrutar su vida laboral bastante. Además, según lo planteado por Moreno-Jiménez et al. (1999), esta dimensión, al igual que la Despersonalización, está más ligada a factores actitudinales del docente que a aspectos organizacionales.

De acuerdo a los resultados arrojados, existe una relación significativa entre esta dimensión y el Estrés de Rol. Esta relación puede estar asociada con que los docentes perciben que las estrategias utilizadas por ellos no son suficientes para enfrentar las demandas laborales, de acuerdo a lo que plantean Matud et al. (2002), Trucco y Valenzuela (1999) y Ponce et al. (2005). Asimismo, tiene que ver con el hecho de que el docente se perciba como si no estuviera desarrollando adecuadamente su trabajo, sintiéndose ineficaz y disminuyendo su realización personal.

En relación con la dimensión de Despersonalización, se encontró que muy pocos de los docentes presentan niveles moderados de este aspecto. Estos hallazgos son contradictorios con lo encontrado en las investigaciones realizadas en Medellín, Ibagué y Bogotá donde se encontró que 34 \%, 55 \% y 30 \% de los docentes de estas ciudades, respectivamente, presentaban niveles positivos de esta dimensión. Por otro lado, estos datos son semejantes a los encontrados por Alderete et al. (2003) y Pando et al. (2006), y podrían estar relacionados con el hecho de que el síndrome es un proceso que se inicia con el desarrollo de ideas sobre fracaso profesional y con actitudes negativas hacia el rol profesional (Falta de Realización Personal en el trabajo), junto a sentimientos de encontrarse emocionalmente agotado (Agotamiento Emocional) y finalmente se generan actitudes y sentimientos negativos hacia las personas con las que se trabaja (actitudes de Despersonalización). Esto de acuerdo al modelo alternativo que proponen Gil-Monte, Peiró y Valcárcel (1998 citados por Gil-Monte, 2000).

Es importante mencionar que en cuanto a las dimensiones de Despersonalización y Falta de Realización Personal no se encontró relación significativa con ningún factor organizacional, lo que es coherente con lo antes mencionado por MorenoJiménez et al. (1999), quienes argumentan que esto puede explicarse por el hecho de que estas dimensiones tienen que ver más directamente con un componente actitudinal que con un componente organizacional, siendo así relevante realizar investigaciones que arrojen explicaciones más amplias sobre aspectos personales que aporten elementos para la explicación de estas dimensiones. 
A manera de conclusión, es importante mencionar que dentro de la investigación se encontró un bajo porcentaje del síndrome de burnout y sus tres dimensiones (Agotamiento Emocional, Despersonalización y Falta de Realización Personal) en los docentes de ambas instituciones (pública y privada). En cuanto a los factores asociados, se encontró relación significativa entre el síndrome de burnout y el Estrés de Rol y los factores organizacionales, al igual que para la dimensión de Agotamiento Emocional. Para la dimensión de Falta de Realización Personal, se encontró una relación con el Estrés de Rol y para la dimensión Despersonalización no se encontró ninguna relación significativa con los factores asociados.

Esta investigación arroja resultados relevantes que dan cuenta de algunos de los factores asociados al síndrome de burnout en la docencia, como el Estrés de Rol, lo cual permite evidenciar la necesidad de intervenir en el desarrollo de estrategias de afrontamiento para el manejo del estrés. Asimismo, es relevante en la medida en que evidencia la importancia de intervenir en factores organizacionales como el estilo y apoyo de la supervisión, condiciones organizacionales y preocupaciones profesionales, dado que esto disminuiría el desarrollo del síndrome.

Es importante mencionar que dentro de la investigación se encontraron algunas limitaciones, que se consideran necesarias para tener en cuenta en la realización de futuras investigaciones. Estas limitaciones tienen que ver, en primer lugar, con la muestra a la cual se le aplicó el cuestionario, pues solo se evaluó una institución para cada tipo de colegio. Adicionalmente, la muestra se seleccionó de forma intencional, lo cual puede haber implicado la introducción de sesgos en los resultados. En segundo lugar, se encontró una limitación en la evaluación referida a la dimensión de Despersonalización, dado que el número de ítems fue insuficiente, y estos indagan directamente aspectos que pueden ir en contra de las expectativas sociales. Adicionalmente, esta subescala ha sido cuestionada en otras investigaciones (MorenoJiménez et al., 2000).
De acuerdo con lo anterior, para futuras investigaciones se recomienda tener en cuenta el aumento de la muestra en relación con el número de colegios de ambos tipos de instituciones y su selección, en lo posible, de manera aleatoria. También se considera necesario destacar las diferencias internas que existen dentro de cada tipo de colegio, por ejemplo, determinar si existen diferencias entre los colegios públicos. Por último, sería recomendable evaluar las propiedades psicométricas del cuestionario para garantizar su validez y fiabilidad.

Otras formas de evaluar el burnout y los factores asociados, podrían ser útiles para contribuir a la comprensión de este fenómeno desde una perspectiva cualitativa.

\section{Referencias}

Albanesi, S., De Bortoli, M. \& Tifner, S. (2006). Aulas que enferman. Psicología y Salud, 2(16), 179-185.

Alderete, M., Pando, M., Arnanda, C. \& Balcázar, N. (2003). Síndrome de burnout en maestros de educación básica, nivel primaria de Guadalajara. Investigación en Salud, 1, 1-10.

Anadón, O. (2005). La formación en estrés para la prevención del síndrome de "burnout" en el curriculum de formación inicial de los maestros. Revista Interuniversitaria de Formación del Profesorado, 19(1), 197-220.

Aranda, C., Pando, M. \& Pérez, M. (2004). Apoyo social y síndrome de quemarse en el trabajo o burnout: una revisión. Psicología y Salud, 1(14), 79-87.

Baró, E. (s. f.). Adolescencia y juventud: concepto y características. Recuperado el 24 de noviembre, 2008, de http://www.sld.cu/libros/libros/libro5/tox1.pdf

Bergadá, M., Neudeck, V., Parquet, C., Tisiotti, P. \& Dos Santos, L. (2005). La salud mental de los educadores: el Síndrome de Burnout en profesionales de una escuela diferencial de la ciudad de Corrientes. Comunicaciones Científicas y Tecnológicas, M-122. Recuperado el 21 de noviembre, 2007, de http:// www.unne.edu.ar/Web/cyt/com2005

Bermejo-Toro, L. \& Prieto-Ursua, M. (2006). Teacher's irracional beliefs and their relationship to distress in the profession. Psychology in Spain, 10(1), 88-96. 
Bórquez, S. (2004). Burnout o estrés circular en profesores. Pharos, 11(1), 23-34.

Byrne, B. M. (1999). The nomological network of teacher burnout: A literature review and empirically validated model. En R. Vandenberghe \& A.M. Huberman (Eds.), Understanding and preventing teacher burnout (pp. 15-37). Nueva York: Cambridge University Press. Recuperado el 18 de junio de 2008, de http://books.google.es/ books?hl=esylr=yid=OrHC3Ny-f_wCyoi=fndyp $\mathrm{g}=\mathrm{PR} 9 \mathrm{ydq}=$ understanding + and + preventing + te achers+burnoutyots $=$ np_k7Er3SPysig $=$ Rbobhn1 HgifBS4TsMcwPYjgW69A\#PPA32,M1

De la Peña, E., Sanz, J., Garrido, J., Carvajal, J., Galan, R. \& Herrero, A. (2002). Factores relacionados con el estrés profesional en personal de enfermería del área de salud de Badajoz. Enfermería Clínica, 12(2), 59-64.

Gil-Monte, P. (2002). Influencia del género sobre el proceso de desarrollo del síndrome de quemarse por el trabajo (burnout) en profesionales de enfermería. Psicologia em Estudo, 7(1), 3-10.

Gil-Monte, P. \& Peiró, J. (1999a). Perspectivas teóricas y modelos interpretativos para el estudio del síndrome de quemarse por el trabajo. Anales de Psicología, 15(2), 261-268.

Gil-Monte, P. \& Peiró, J. (1999b). Validez factorial del Malasch Burnout Inventory en una muestra multidimensional. Psicothema, 11(3), 679-689.

Hermosa, A. (2006). Satisfacción laboral y síndrome de "burnout" en profesores de educación primaria y secundaria. Revista Colombiana de Psicología, 15, 81-89.

Hernández, G. L. \& Olmedo, E. (2004). Un estudio correlacional acerca del síndrome de "estar quemado" (burnout) y su relación con la personalidad. Apuntes de Psicología, 22(1), 121-136.

Hernández, G. L., Olmedo, E. \& Ibáñez, I. (2004). Estar quemado (burnout) y su relación con el afrontamiento. Internacional Journal of Clinical and Health Psychology, 4(2), 323-336.

Hernández-Sampieri, R., Fernández, C. \& Baptista, P. (1998). Metodología de la investigación. México: McGraw-Hill Interamericana.
Maslach, C. (2002, agosto). Job burnout: New directions in research and intervention. Presentation at American Psychological Association Convention, Chicago, United States.

Matud, M. P., García, M. \& Matud, M. J. (2002). Estrés laboral y salud en el profesorado: un análisis diferencial en función del género y del tipo de enseñanza. Revista Internacional de Psicología Clínica y de la Salud, 2(3), 451-465.

Moreno, M., Aranda, C., Aldrete, M. G., Flores, E. \& Pozo, E. (2006). Factores psicosociales y burnout en docentes del centro universitario de ciencias de la salud. Investigación en Salud, 7(3), 173-177.

Moreno-Jiménez, B., Arcenillas M., Morante, M. \& Garrosa, E. (2005). Burnout en profesores de primaria: personalidad y sintomatología. Revista de Psicología del Trabajo y las Organizaciones, 21, 71-91. Moreno-Jiménez, B., Garrosa, E. \& González, J. (1999). Burnout docente, sentido de coherencia y salud percibida. Revista de Psicopatología y Psicología Clínica, 4(3), 163-180.

Moreno-Jiménez, B, Garrosa, E. \& González, J. (2000). La evaluación del estrés y el burnout del profesorado: el CBP-R. Revista de Psicología del Trabajo y de las Organizaciones, 16(1), 331 -349.

Moreno-Jiménez, B. \& Peñacoba, C. (1999). Estrés asistencial en los servios de salud. En M. A. Simón (Ed.), Manual de psicología de la salud (pp. 739-764). Madrid: Biblioteca Nueva.

Moriana, J. \& Herruzo, J. (2004). Estrés y burnout en profesores. International Journal of Clinical and Health Psychology, 4(3), 597-621.

Olmedo, M., Santed, M. A., Jiménez, R. \& Gómez, M. D. (2001). El síndrome de burnout: variables laborales, personales y psicopatológicas asociadas. Psiquis, 22(3), 117-129.

Ortega, C. \& López, F. (2003). El burnout o síndrome de estar quemados en los profesionales sanitarios: revisión y perspectiva. International Journal of Clinical and Health Psychology, 4(1), 137-160.

Pando, M., Aranda, C., Alderete, M., Flores, E. \& Pozo, E. (2006). Factores psicosociales de burnout en docentes del centro universitario de ciencias de la salud. Investigación en Salud, 8(3), 173-177. 
Ponce, C. R., Bulnes, M. S., Aliaga, J. R., Atalaya, M. C. \& Huertas, R. E. (2005). El síndrome del "quemado" por estrés laboral asistencial en grupos de docentes universitarios. Revista IIPsi, 8(2), 87-112.

Restrepo-Ayalal, N., Colorado, G. \& Cabrera, G. (2005). Desgaste emocional en docentes oficiales de Medellín. Revista de Salud Pública, 8(1), 63-73.
Tisiotti, P., Parquet, C. \& Neudeck, V. H. (2007). Prevalencía y dimensiones del burnout en profesionales de una escuela diferencial de la ciudad de Corrientes. Revista de Postgrado de la Vía Cátedra de Medicina, 172, 4-7.

Trucco, M. \& Valenzuela, P. (1999). Una batería de cuestionarios para el estudio de estrés ocupacional. Revista Científica Chilena, 34-76. 
